\title{
Quadcopter Based Object Detection and Localization
}

\author{
Malik A. Alsaedi ${ }^{1}$, Baraa M. Albaker ${ }^{2}$, Hussein Ali Dawood ${ }^{3}$, \\ Haider abd aoun ${ }^{4}$, Zeyad kamel tayeh ${ }^{5}$ \\ ${ }^{1}$ College of Engineering /Al-Iraqia University \\ maliksaady@yahoo.com \\ ${ }^{2}$ College of Engineering, Al-Iraqia University \\ baraamalbaker@ymail.com \\ ${ }^{3}$ College of Engineering,/Al-Iraqia University \\ hussien.ali14@gmail.com \\ ${ }^{4}$ College of Engineering/Al-Iraqia University \\ haideraltemiy@gmail.com \\ ${ }^{5}$ College of Engineering/Al-Iraqia University \\ ziadalzubaee@gmail.com
}

\begin{abstract}
This paper presents the development and integration of an X-configuration quadcopter with an IP camera for object detection based on the color of an object. A KK2 microcontroller is used to control the quadcopter movements. The controller has builtin gyros that provide heading rate information, which are used to control the movement of the quadcopter. The parts of the whole UAV are selected and integrated. The calibration processes are handled after installing necessary controller codes. This is to make quadcopter fly smoothly according to its set commands. The IP camera is then attached to the quadcopter frame. Mobile camera with GPS receiver is used for object detection and localization. Images are captured from the IP camera; the three-dimensional position information for the quadcopter is recorded by the GPS receiver, and then the whole data are sent repeatedly from the Mobile to a ground station computer via wireless communication link. Two algorithms are next implemented to acquire and analyze the Images received form the IP camera. The results show that the images was captured and analyzed successfully, and the objects were detected according to their color and its approximate position was reported.
\end{abstract}

\section{INTRODUCTION}

Unmanned Aerial Vehicles (UAVs) are typically commissioned for tasks that are perceived as too dull or dangerous for a human pilot to carry out. In the past, research involving UAVs was constrained by large and expensive flight platforms which offered greater payloads. However, recent advances in embedded computing and sensors have made small, low-cost autonomous systems accessible to the broader research community [1]. These little flying robots are utilized for investigation of sun oriented boards and furthermore connects. What's more, they can aid protect missions after catastrophic events or blasts, or screen untamed life and product. They can likewise supplant people amid exercises that could be destructive to life, for example, surveillance in ranges with an abnormal state of radiation[2]. Unmanned Aerial Vehicles (UAVs) have as of late excited extraordinary interests in both mechanical and military fields. UAV is a kind of extremely complex framework which coordinates diverse equipment segments, for example, camera, Global Positioning System (GPS), controller, and distinctive programming segments, for example, picture preparing, way arranging and internal circle control[3]. A preliminary hierarchy architecture that describes system levels and sensors and actuators with an autopilot control example can be found in [4-6]. Ethereal vehicle frameworks have particular qualities like flying in low heights and having the capacity to drift that make them reasonable for applications that might be difficult to finish utilizing settled wing vehicles[7]. It merits saying that regardless of the way that the quadrotor structure and its weight are consistent however its payload can be changed in different applications. So a flexible criticism control is fundamental to have an $\mathrm{OK}$ execution in spite of various introductory conditions and different circumstances amid flight [8]. Generally it turns to be even more complicated for rotorcraft designs. However pose a number of advantages compared to the fixed wing systems for a wide set of reallife applications, such as a) hovering ability, b) higher payloads, and c) aggressive maneuverability[9]. Cameras have the advantages of being minimal effort, low power, little size and light weight, and the capacity of being utilized for different errands, for example, for exploring and also performing reconnaissance [10]. In this project we consider a single UAV with a bird's-eye by use smart phone as IP camera (Huawei phone) to get images for object and detect object position by using GPS technique of the smart phone. We begin by proposing a target detection algorithm that determines whether a target protest exists in a video outline. One would expect that the viability of such a classifier changes as indicated by its shades of the objective.

\section{WORK METHODOLOGY}

Several steps are performed here to develop the quadcopter, and to capture and analyze images and GPS data (See Fig. 1). The first step, after studying several types of UAVs models which are used for different purposes, is to select right components for the development process of the quadcopter. The appropriate parts, including low-level controller that controls the UAV movement, UAV frame, motors, Electronic Speed Controllers (ESCs), brushless DC motors, propellers, and battery size and power which are carefully selected to overcome demanding power by whole UAV's aviation electronics, are selected. Later, individual UAV parts are integrated and low-level UAV control program is downloaded to the controller. After that, the testing and Calibration process are invoked to test the 
direction of propellers according to predefined direction and calibrate the equipment, which further includes calibration of ESCs, motors, and microcontroller.

Finally, after integrating and flight testing the developed quadcopter, we used mobile as a camera attached with other components of the quadcopter. This is to act as an online images recorder and a global Positioning System (GPS) receiver. Two algorithms are developed next to capture online images and extract GPS position information defined by altitude, latitude and longitude. The second developed algorithm is to upload the captured images and on-line detect objects based on their colors. At the end, UAV flight are tested in different flight modes and several images were captured and analyzed to show strength of the developed algorithms to detect objects successfully.
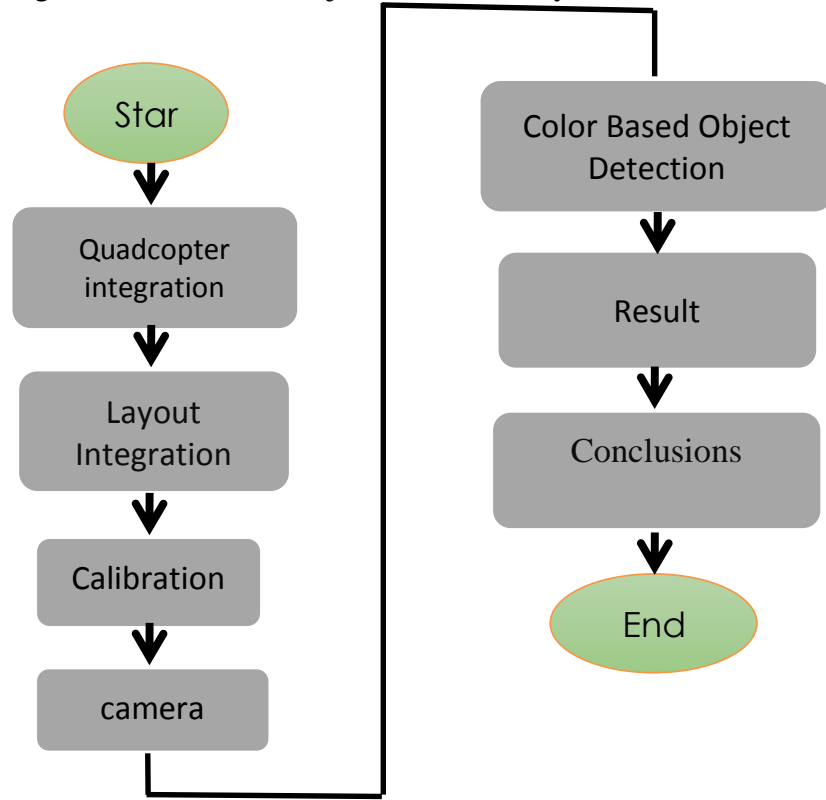

Figure 1: The steps carried out in this paper.

\section{QUAD-COPTER STEPS}

INTEGRATION

The appropriate and required parts which selected to complete quadcopter are Microcontroller ,four electronic speed control "ESCs", four motors each motor $1000 \mathrm{KV}$, four Propellers and Radio Controller RC “ Transmitter” and Receiver .

After that connected and integrated the quadcopter parts, first Connected electronic speed control "ESCs" to the frame board and microcontroller from first side ,other side of ESCs connect to the motors, then Connected the motors on the arms of quadcopter, and any two motors in same axis must rotate in same direction and reverse other two motors in other axis as shown in Fig. 2, then connect ESCs and RC Receiver to Microcontroller after complete programming it. quadcopters utilizes four propellers and four engines which makes push and lifts high, out of four engines with propellers connected, two engines turns in clockwise $(\mathrm{CW})$ and other two pivots in counter-clockwise(CCW). As appeared in Fig. 2, Motors 1 and 3 ought to turn in clock astute while engines 2 and 4 ought to pivot in counter clock shrewd heading.

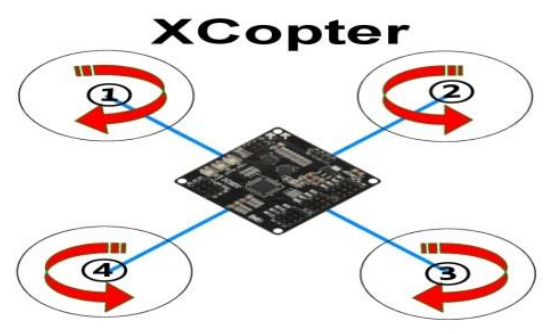

Figure 2: Propellers rotations of the developed xconfiguration quadcopter

\section{LAYOUT OF THE INTEGRATED QUADCOPTER}

The integrated UAV system consists of a microcontroller which is considered as the heart of quadcopter that controls the movements of the quadcopter. It is also used to stabilize and balance the UAV using its gyros and accelerometers sensors. The microcontroller is connected with a RC receiver, which receives guidance information received from RC Transmitter and passes them to microcontroller and accordingly the controller changes movement of quadcopter by controlling the speed of motors that use ESCs, ESCs function is control by speed of motors. Fig. 3 shows the basic layout overview of the developed quadcopter, while Fig. 4 depicts the developed UAV after the integration process.

A mobile system is also integrated to the developed UAV. It is used as camera for online imaging purpose and GPS information extractor. The advantage of using mobile as compared with other platforms includes: high image resolution, GPS information provider, less weight, and more battery life

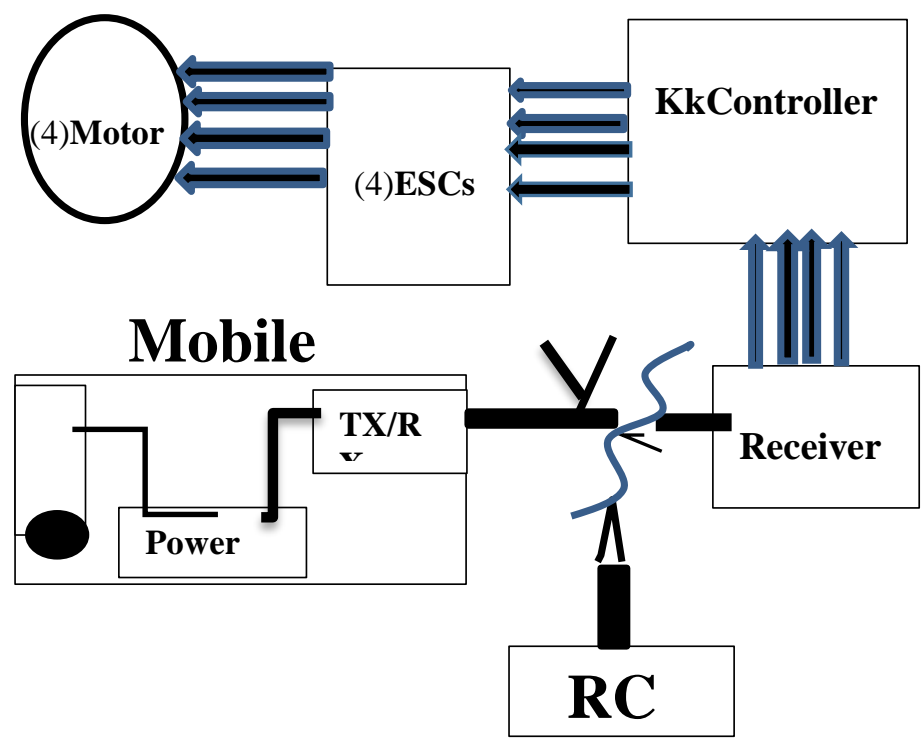

Figure 3: Basic layout of the overall Quadcopter 


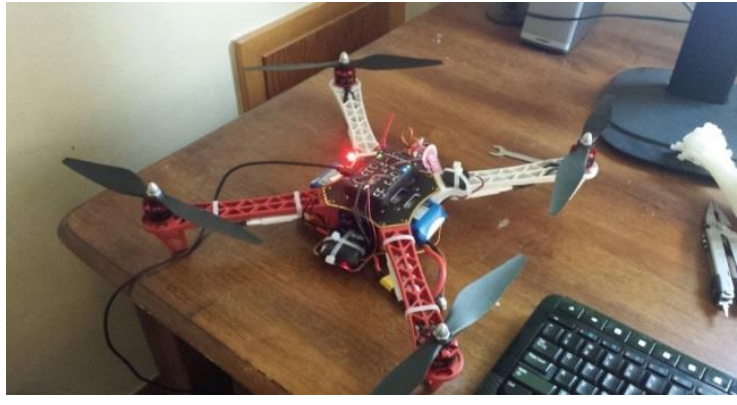

Figure 4: Developed Quadcopter after integration

\section{QUADCOPTER OPERATION AND CONTROL}

The RC Transmitter is used to control quadcopter movements in different modes and directions: up, down, left and right movement of quadcopter. These movements can be achieved by changing the speed of motors.

First, Yaw movement, also called rudder, is the deviation the head of the drone either to left or right as demonstrated in Fig. 5. The bold green mean more thrust and quadcopter will move to right angle.

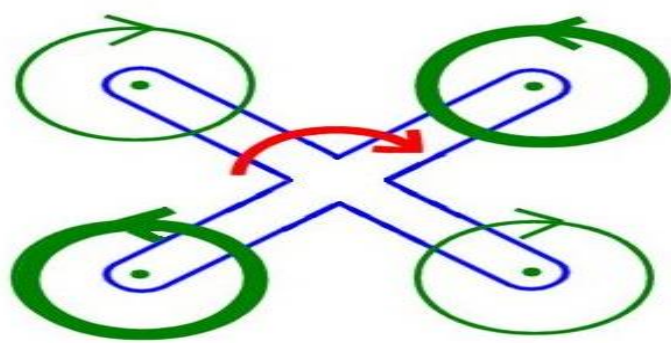

Figure 5: Propellers spin demonstration for Roll control

Second, the Pitch movement allows movingthe quadcopter either forward or in reverse. Forward Pitch is accomplished by pushing the aileron stick in RC Transmitter forward, which makes the quadcopter tilt and advance. In reverse pitch is accomplished by moving the aileron stick in RC Transmitter in reverse, as demonstrated in Fig. 6.

Pitch Down

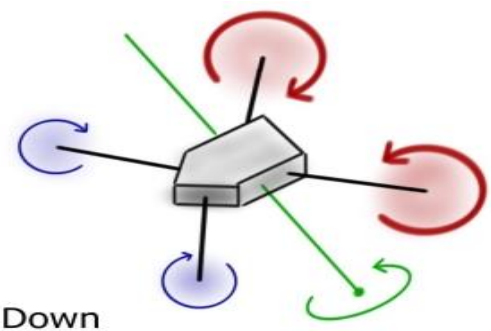

Figure 6: Propellers spin demonstration for Pitch control

Third, move development is making the quadcopter fly sidewards, either to left or right. Roll is controlled with the aileron stick, making it move left of right. on the off chance that the aileron stick moved to one

side, the quadcopter will fly left, while if the aileron stick moved to right, the quadcopter will fly right (See Fig. 7).

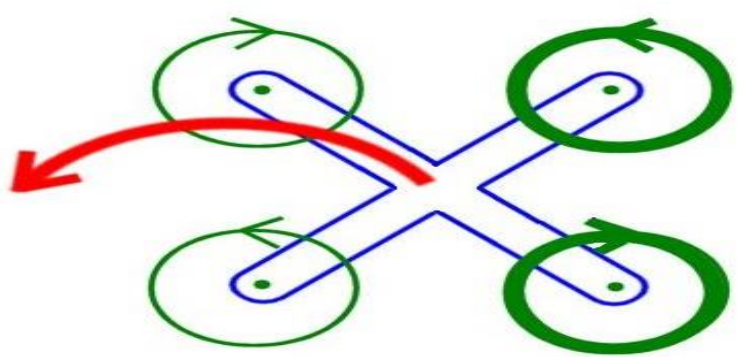

Figure 7: Propellers spin demonstration for Roll control

\section{6-CALIBRATION OF MICROCONTROLLER: L}

The calibration process was initiated after integrating the whole UAV. It involves many calibration sub-processes that must be done before the real deployment of the UAV. This is to insure smooth UAV flying according to the instructed commands sent by the RC transmitter. The first calibration is to arm and disarm the flight-controller that insures safety feature which disables the throttle stick. The second calibration sub-process is to calibrate the throttle range on the UAV's ESCs. This is to ensure that all the ESC's have the same throttle range. The third calibration is to check the direction of the transmitter channels. This step is to ensure that the sticks actually perform the action in the way that they are supposed to operate. The fourth calibration subprocess is to check the gyro compensations. This step is to ensure that the gyros compensate in the right direction. If they don't, the UAV will be uncontrollable. The last include reversing the pots direction. This calibration is required if the pots turn in the wrong direction.

\section{7- CAMERA FOR DETECTING OBJECTS BY ITS COLOR}

In this project, we use a smart phone (called Huawei phone) as IP camera to get images for object and detect UAV position by using GPS facility built in the smart phone.

Wi-Fi technique is used to connect devices wirelessly by a router, and to get on-line images from smart phone. we use droidcam application for this purpose as shown in Fig.8 below..

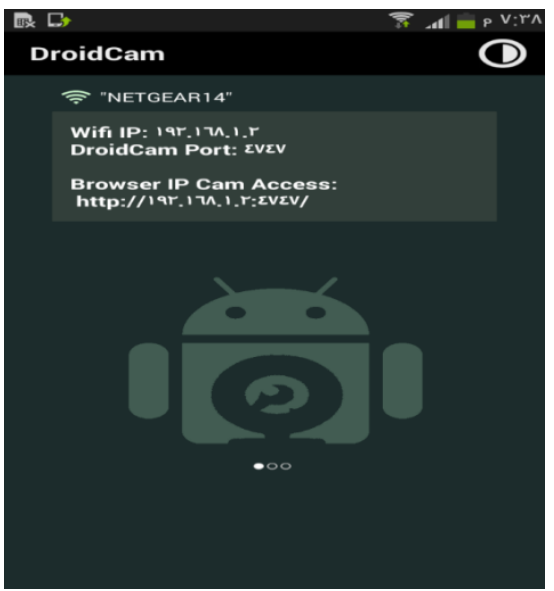

Figure 8: Droidcam application used to acquire images 


\section{8- RESULTS}

A visual studio program was developed to act as a user interface to show acquired images before and after objects detection. Another window was created to display GPS 3D position information on google maps. We have created a special code to identify a particular color and cancel the rest of the colors by giving them a specific color. As an example, white color to distinguish the chosen color from the rest of colors as illustrated in Fig. 9. The GPS position information was repeatedly recorded using the real-time GPS tracker application that would work online with internet to detect UAV position as depected in Fig. 10.

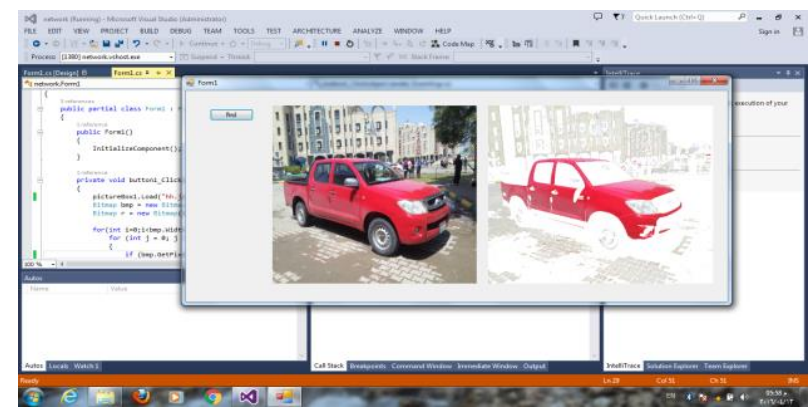

Figure 9: Illustrates an object detection based on red color

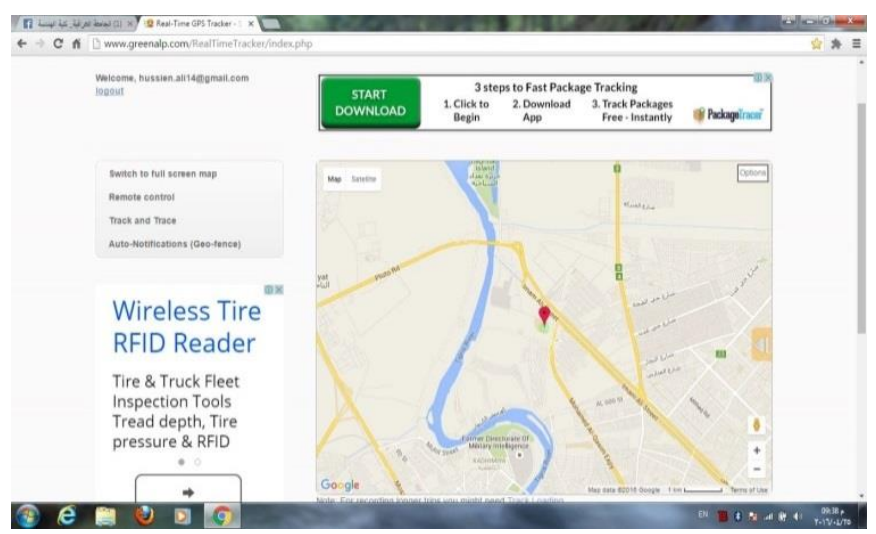

Figure 10: Illustrates GPS real-time positioning on google maps for UAV

\section{9- CONCLUSION}

This paper addresses the problem of how to develop and integrate a quadcopter. The work also involves object detection based on its color. Quadcopter position was recorded repeatedly with the acquired images to give more information about the approximate location of the detected object. Several steps were handled in the integration and calibrations processes of the developed UAV. The identification of the color is based on quantity (brightness) of color of the object that needs to be detected. Other colors in the image will be excluded and discarded, except the specified one. Using GPS facility built on smart phone application (Real-Time GPS tracker), the
UAV 3D position specified by longitude, latitude, and altitude will be obtained. Thus, online images acquisition of objects with a 3D UAV position will be recorded, displayed and analyzed for object detection on our developed user interface and image detection programs. The results show the successful implementation of the developed algorithms

\section{REFERENCES}

[1] H. Liu, Y. Bai, G. Lu, Z. Shi, and Y. Zhong, "Robust Tracking Control of a Quadrotor Helicopter," $J$. Intell. Robot. Syst., vol. 75, no. 3-4, pp. 595-608, 2014.

[2] A. Chovancová, T. Fico, L. Chovanec, and P. Hubinsk, "Mathematical Modelling and Parameter Identification of Quadrotor (a survey)," Procedia Eng., vol. 96, pp. 172-181, 2014.

[3] Y. Bi and H. Duan, "Implementation of autonomous visual tracking and landing for a low-cost quadrotor," Opt. - Int. J. Light Electron Opt., vol. 124, no. 18, pp. 3296-3300, 2013.

[4] B. M. Albaker, "Preliminary Architectonic Design for a Smart Solar-Powered UAV," IEEE Conference on Clean Energy and Technology (CEAT 2013), Bayview Hotel, Langkawi, Malaysia, 18-20 Nov. 2013.

[5] B. M. Albaker, N. A. Rahim, "Design of an Autonomous Unmanned Aircraft Flight Path Controller," In the proceedings of the IEEE International Conference on Electrical Control and Computer Engineering (INECCE 2011), Kuantan, Malaysia, pp. 229-232, 21-22 June 2011.

[6] B. M. Albaker and N. A. Rahim, "Flight Path PID Controller for Propeller-Driven Fixed-Wing Unmanned Aerial Vehicles," International Journal of Physical Sciences, Vol. 6, No.8, pp. 1947-1964, April 2011.

K. Valvanis, "Advances in unmanned aerial vehicles," Intell. Syst. Control. Autom. Sci., pp. 171210, 2007.

[8] G. M. Hoffmann, H. Huang, S. L. Waslander, and C. J. Tomlin, "Quadrotor Helicopter Flight Dynamics and Control : Theory and Experiment," Am. Inst. Aeronaut. Astronaut., vol. 4, no. August, pp. 1-20, 2007.

[9] K. Alexis, G. Nikolakopoulos, and A. Tzes, "Experimental constrained optimal attitude control of a quadrotor subject to wind disturbances," Int. J. Control. Autom. Syst., vol. 12, no. 6, pp. 1289-1302, 2014.

[10] V. Ghadiok, J. Goldin, and W. Ren, "On the design and development of attitude stabilization, visionbased navigation, and aerial gripping for a low-cost quadrotor," Auton. Robots, vol. 33, no. 1-2, pp. 4168, 2012. 